\title{
Localization of the equine lgG-binding domain in the fibrinogen-binding protein (FgBP) of Streptococcus equi subsp. equi
}

Correspondence
Mary Meehan
meehanmary@yahoo.co.uk

Received 4 March 2009

Revised 24 April 2009

Accepted 6 May 2009

\author{
Mary Meehan, ${ }^{1} \dagger$ Melanie J. Lewis, ${ }^{2}$ † Caroline Byrne, ${ }^{1}$ David O'Hare, ${ }^{1}$ \\ Jenny M. Woof ${ }^{2}$ and Peter Owen ${ }^{1}$
${ }^{1}$ Department of Microbiology, Moyne Institute of Preventative Medicine, Trinity College, Dublin 2, Ireland DD1 9SY, UK \\ ${ }^{2}$ Division of Medical Sciences, University of Dundee Medical School, Ninewells Hospital, Dundee
}

\begin{abstract}
Fibrinogen-binding protein (FgBP, also termed SeM) is a cell-wall-associated anti-phagocytic Mlike protein of the equine pathogen Streptococcus equi subsp. equi, and binds fibrinogen (Fg) and IgG. FgBP binds $\mathrm{Fg}$ avidly through residues located at the extreme $\mathrm{N}$ terminus of the molecule, whereas the IgG-binding site is more centrally located between the $A$ and $B$ repeats. FgBP binds equine $\operatorname{lgG} 4$ and $\lg$ 7 7 subclasses through interaction with the $\mathrm{CH} 2-\mathrm{CH} 3$ interdomain region of IgG-Fc, and possesses overlapping Fc-binding sites with protein A and protein $\mathrm{G}$. In this study, FgBP truncates containing defined internal deletions were used to identify a stretch of 14 aa (residues 335-348) critical for lgG binding. Protein chimeras consisting of the non-lgG-binding $\alpha$-helical coiled-coil M5 protein fused to FgBP sequences were used to identify a minimal equine IgG-binding domain consisting of residues 329-360. Competition ELISA tests suggested that IgG does not compromise Fg binding and vice versa.
\end{abstract}

\section{INTRODUCTION}

Streptococcus equi subsp. equi (herein referred to as $S$. equi) is the causative agent of strangles, a highly contagious, debilitating disease of the upper respiratory tract and associated lymph nodes of the horse (Timoney, 1993). S. equi is an obligate pathogen of the family Equidae, and is one of the most prevalent diseases of the horse, causing serious economic loss. With no adequate vaccine, prevention relies on prompt identification of infected horses, in combination with isolation and hygiene control measures (Waller \& Jolley, 2007).

S. equi possesses many secreted and surface-associated proteins which bind host serum or matrix proteins and are likely to play roles in adhesion, invasion and evasion of the immune response (reviewed by Timoney, 1993, 2004; Harrington et al., 2002; Waller \& Jolley, 2007). These include two fibrinogen $(\mathrm{Fg})$-binding proteins (SzPSe and $\mathrm{M}$ protein). Of these, the $\mathrm{M}$ protein is considered to be an important virulence determinant (Meehan et al., 1998, 2001; Timoney et al., 1997). Fifteen alleles of the gene that encodes M protein have so far been identified (Kelly et al., 2006; Waller \& Jolley, 2007). M protein encoded by strain

†These authors contributed equally to this work.

Abbreviations: Fg, fibrinogen; FgBP, fibrinogen-binding protein; HRP, horseradish peroxidase; eqFg, equine $\mathrm{Fg}$; eqlgG, equine lgG.
TW and termed FgBP (fibrinogen-binding protein; gene allele 1) in our previous publications is almost identical to the prototype $\mathrm{M}$ protein (termed SeM) of strain CF32 (gene allele 2; Meehan et al., 1998; Timoney et al., 1997). The term FgBP will be retained in this publication to facilitate correlation with our previous publications and truncates (Lewis et al., 2008a; Meehan et al., 1998, 2000a, b, 2001, 2002).

$\mathrm{FgBP}$ possesses sequence and structural features in common with the cell-wall-associated $\mathrm{M}$ proteins of Streptococcus pyogenes, including a high proportion (over $60 \%$ ) of coiled-coil dimeric structure and Fg-binding ability (Fig. 1a). The region of FgBP involved in Fg binding is localized in the extreme $\mathrm{N}$ terminus of the protein, but requires downstream stabilizing coiled-coil structure (Meehan et al., 2000a, b). There is convincing evidence that FgBP plays a significant role in resistance to phagocytosis and that the Fg-binding region is involved in this process (Boschwitz \& Timoney, 1994a, b; Chanter et al., 2000; Meehan et al., 1998, 2001). The observed variability among the $\mathrm{N}$ termini of $\mathrm{FgBP}$ proteins from different $S$. equi strains has been exploited as a tool in epidemiological studies (Anzai et al., 2005; Kelly et al., 2006; Waller \& Jolley, 2007).

FgBP additionally binds to the $\mathrm{CH} 2-\mathrm{CH} 3$ interdomain region of equine $\operatorname{IgG}$ (eqIgG)-Fc (Lewis et al., 2008a; 


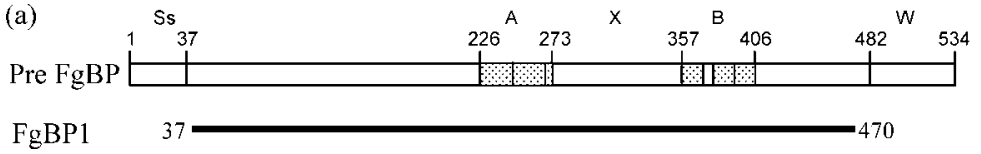

(b)
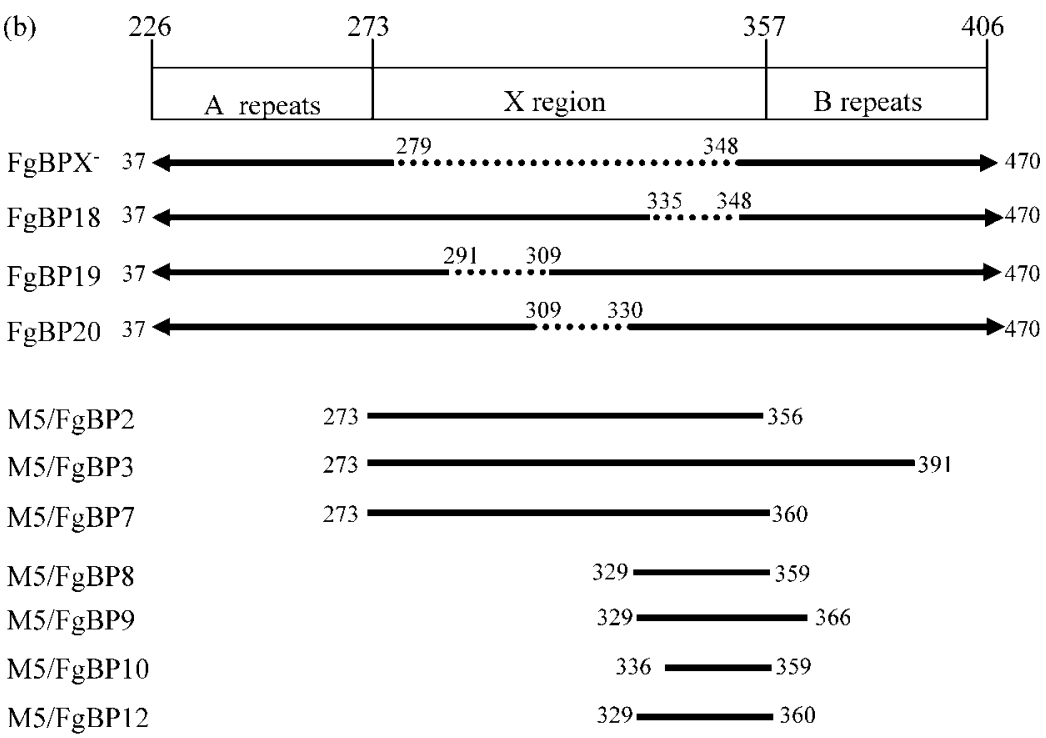

(c)

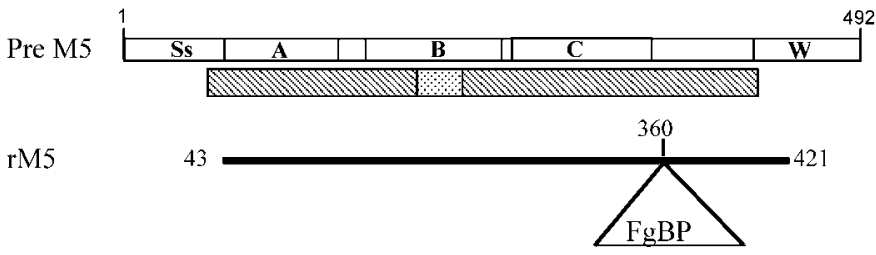

Fig. 1. Schematic diagram of (a) FgBP, (b) His-tagged recombinant FgBP truncates and M5FgBP chimeric proteins, and (c) M5 protein. (a) The positions of the signal sequence (Ss), $A$ and $B$ repeat domains, the $X$ region between the $A$ and $B$ repeats, and the wall/ membrane (W)-spanning region in unprocessed FgBP are indicated. Below the structure of pre-FgBP is a scaled representation of the recombinant His-tagged FgBP1 protein, which exhibits wild-type FgBP binding to both $\mathrm{Fg}$ and $\lg \mathrm{G}$. (b) Below a schematic diagram of the central region of FgBP are scaled representations of FgBP truncates constructed in this study. Dotted lines indicate the regions deleted. Also shown are scaled representations of the regions of FgBP which were used to construct M5FgBP chimeric proteins. (c) The positions of the signal sequence (Ss), the repeat regions ( $A, B$ and $C$ ) and the wall/ membrane (W)-spanning domain of M5 protein are indicated. The box below indicates the regions of M5 predicted to have $90-100 \%$ (hatched) and 22-62\% (dotted) $\alpha$-helical coiled-coil structure. Also shown is a scaled representation of the recombinant M5 protein indicating the region into which FgBP sequences were inserted.
Meehan et al., 2001). This is one of the few regions in IgG particularly suited for protein-protein interaction, and numerous bacterial, viral and host proteins bind to this region (Burton, 1985; DeLano et al., 2000). Most bacterial immunoglobulin-binding proteins exhibit little or no sequence homology and are generally considered to have converged during evolution to bind overlapping sites in the interdomain region, with the observed diversity in immunoglobulin-binding profiles with respect to isotype, subclass and species being accounted for by the different binding interactions (Frick et al., 1992; Goward et al., 1993; Pleass et al., 2001). The best studied IgG-binding proteins are protein A and protein G. Protein G and protein G-like proteins are responsible for much of the IgG binding by group C and group G streptococci (Björck et al., 1987; Navarre \& Schneewind, 1999), whereas IgG binding by Staphylococcus aureus is mediated by protein A and/or Sbi, a protein A-like protein (Atkins et al., 2008; Moks et al., 1986; Uhlén et al., 1984). In S. pyogenes, immunoglobulin binding is mediated by three groups of $\mathrm{M}$ proteins encoded by the emm, mrp and enn genes.
Recently, Lewis et al. (2008a) characterized the immunoglobulin-binding properties of FgBP and showed that of the seven equine IgG subclasses, FgBP binds predominantly to IgG4 and IgG7. Together, IgG4 and IgG7 form the originally designated eqIgGb subclass, which is the most common IgG subclass in the serum and secretions of horses (Sheoran et al., 1997). FgBP has been shown to require both the $\mathrm{CH} 2$ and the $\mathrm{CH} 3$ domain of $\mathrm{IgG}-\mathrm{Fc}$ for full binding, the binding site exhibiting significant overlap with the Fc-binding sites for protein $A$ and protein $G$ (Lewis et al., 2008a). Significantly, the interaction of FgBP with IgG is able to disrupt $\mathrm{Clq}$ binding and to inhibit the antibody-mediated classical complement pathway, suggesting another mechanism by which FgBP mediates immune evasion (Lewis et al., 2008a).

Previous studies utilizing a series of FgBP truncates clearly showed that the binding of $\mathrm{Fg}$ and $\operatorname{IgG}$ requires distinct structural features (Meehan et al., 2000a, b, 2001). IgG binding does not involve the extreme $\mathrm{N}$ terminus of FgBP required for Fg binding but rather a region spanning the 
central portion of the protein between the $\mathrm{A}$ and $\mathrm{B}$ repeats (Meehan et al., 2001). In this report, recombinant FgBP proteins possessing internal deletions and $\mathrm{M} 5 \mathrm{FgBP}$ chimeric proteins were constructed and were employed to further define the IgG-binding site. Results suggest that IgG binding is mediated by a stretch of amino acids (329-360) adjacent to the $\mathrm{B}$ repeats. Within this 32-residue domain is a stretch of 14 aa shown to be critical for ligand binding.

\section{METHODS}

Bacterial strains, plasmids and growth conditions. Escherichia coli XL1-Blue (Stratagene) and plasmid pQE30 (Qiagen) were used for expression of recombinant $\mathrm{FgBP}$ and M5 proteins. E. coli strains were grown at $37{ }^{\circ} \mathrm{C}$ in LB medium supplemented where appropriate with ampicillin $\left(100 \mu \mathrm{g} \mathrm{ml}^{-1}\right)$ and $1 \mathrm{mM}$ IPTG. Plasmid pLZM5 was used as a template for PCR amplification of the S. pyogenes M5 gene (Ringdahl et al., 2000; Johnsson et al., 1998). S. equi subsp. equi strain TW genomic DNA and pQE30-FgBP1 were used as templates for PCR of $f b p$.

Recombinant DNA techniques. Genomic DNA was isolated from $S$. equi using the Edge BioSystems bacterial genomic DNA purification kit. Plasmid DNA was purified from E. coli using the Promega Wizard plasmid purification kit. DNA restriction digestions, ligations and transformations were carried out by standard methods (Sambrook et al., 1989).

Expression and purification of recombinant proteins. FgBP1 was produced as previously described (Meehan et al., 1998). Recombinant FgBP proteins containing internal deletions were constructed by amplification of $f b p$ DNA fragments flanking the region to be deleted followed by their subsequent ligation in the expression plasmid pQE30 (Fig. 1b). pQE30-M5 was constructed by ligation of amplified DNA encoding amino acids $43-421$ of the M5 protein into pQE30 predigested with BamHI and XmaI. pQE30-M5FgBP recombinant plasmids were generated by (i) inverse PCR of pQE30-M5 DNA at nucleotide 1122 of the M5 gene using primers with SacI and EcoRV restrictions sites and (ii) ligation of amplified $f b p$ DNA fragments into amplified pQE30-M5 DNA. His-tagged recombinant FgBP and M5 proteins were purified from E. coli XL-1 Blue by metal chelate affinity chromatography, as described previously in detail (Meehan et al., 1998 , 2000a). Primers used in the construction of the FgBP/M5 recombinant proteins are listed in Table 1. Recombinant eqIgG subclasses were produced and purified as previously described (Lewis et al., 2008b).

Biochemical procedures. SDS-PAGE was performed using either 12.5 or $7.5 \%(\mathrm{w} / \mathrm{v})$ polyacrylamide separating gels and a $4.5 \%(\mathrm{w} / \mathrm{v})$ polyacrylamide stacking gel. Samples were routinely heated for $3 \mathrm{~min}$ at $100{ }^{\circ} \mathrm{C}$ in Laemmli sample buffer prior to electrophoresis (Laemmli, 1970). Proteins were detected by staining with Coomassie brilliant blue. Molecular masses were determined from the relative mobilities of 15 standard molecular mass marker proteins (Gibco-BRL, BenchMark protein ladder). Protein concentration was estimated by the method of Bradford (1976) using BSA as a standard.

Immunological and affinity procedures. eqIgG-Fc affinity immunoblotting was performed as described previously (Meehan et al., 2001) using horseradish peroxidase (HRP)-conjugated horse Fc (10 $\mu \mathrm{g} \mathrm{ml}^{-1}$; Jackson Immunoresearch). Fg-affinity blotting using HRP-labelled equine $\mathrm{Fg}$ (eqFg) was performed as described previously (Meehan et al., 1998, 2000a). Prior to labelling with HRP, contaminating IgG was firstly removed from eqFg (Sigma Aldrich) using protein $\mathrm{G}$ agarose (Sigma Aldrich).
Table 1. PCR primers used to construct recombinant FgBP and M5 proteins

\begin{tabular}{|ll|}
\hline Primer & \multicolumn{1}{|c|}{ Sequence $\left(\mathbf{5}^{\prime}-\mathbf{3}^{\prime}\right) \dagger$} \\
\hline F1 & \multicolumn{1}{|c|}{ cgcggatccaactctgaggttagtcgtacg } \\
R1 & atctgctgttttagctgcc \\
F2 & ccgggatatcgaatcaggcaaacgtgaaattgc \\
R2 & gcgcceggttattgcttcaagcgattgatttc \\
F3 & atcactgctgataaggctaaggc \\
R3 & atctgtttgaagtgatgcaatggtg \\
F4 & atccaaacagagctagaaaaagc \\
R4 & ccgggatatctagactaccaacgtttc \\
F5 & cgcggatccgccgtgactaggggtacaataaatg \\
R5 & ccgggatatcaatttctgctaattttgagc \\
F6 & cggcgagctcaaattagctgctcttgaaaac \\
R6 & cgcccegggggtttgtgagtctgatgcttttgg \\
F7 & cggcgatatcgcagctaaaacagcagaagc \\
R7 & cggcgatatcgctgtttgcttcttctaaagc \\
F8 & cggcgatatcgcatcacttcaaacagagctag \\
R8 & cggcgagctcaatttcacgtttgcctgattc \\
F9 & cggcgatatcgaaaaagctaagacagagcttg \\
R9 & cggcgagctctaccttagcatctgatgctgc \\
R10 & cagggagctcttgtagctcagcaatttc \\
R11 & cagggagctctagctcagcaatttcacgttg \\
R12 & cagggagctcagaagcatcttttgtttttg \\
& \\
\hline
\end{tabular}

*The combinations of primers used in construction of recombinant proteins were: F1 with R1 and F2 with R2 $\left(\mathrm{FgBPX}^{-}\right)$; F1 with $\mathrm{R} 3$ and F2 with R2 (FgBP18); F1 with R4 and F3 with R2 (FgBP19); F1 with R5 and F4 with R2 (FgBP20); F5 with R6 (M5); F6 with R7 (pQE30M5 inverse PCR); F7 with R8 (M5FgBP2); F7 with R9 (M5FgBP3); F7 with R10 (M5FgBP7); F8 with R11 (M5FgBP8); F8 with R12 (M5FgBP9); F9 with R11 (FgBP10); F8 with R10 (M5FgBP12). $\dagger$ Restriction sites are underlined.

ELISAs to test binding to recombinant eqIgG4 and eqIgG7 were performed as previously described (Lewis et al., 2008a). Plates (96well) were coated with recombinant FgBP proteins $\left(10 \mu \mathrm{g} \mathrm{ml}^{-1}\right)$, probed with eqIgG4 or eqIgG7 $\left(0-20 \mu \mathrm{g} \mathrm{ml}^{-1}\right)$ in PBS $(140 \mathrm{mM} \mathrm{NaCl}$, $2.7 \mathrm{mM} \mathrm{KCl}, 10 \mathrm{mM} \mathrm{Na}_{2} \mathrm{HPO}_{4}, 1.8 \mathrm{mM} \mathrm{KH}_{2} \mathrm{PO}_{4}, \mathrm{pH}$ 7) containing $0.5 \%(\mathrm{v} / \mathrm{v})$ Tween-20 (PBS-T) followed by HRP-conjugated goat antimouse $\lambda$ light-chain antibody $\left(0.2 \mu \mathrm{g} \mathrm{ml}{ }^{-1}\right.$ in PBS-T; Bethyl Laboratories).

For competition ELISA tests, 96-well plates were coated overnight at $4{ }^{\circ} \mathrm{C}$ with $\operatorname{FgBP}\left(0.25 \mu \mathrm{g} \mathrm{ml}^{-1}\right)$, washed and then blocked with $5 \%$ $(\mathrm{w} / \mathrm{v})$ non-fat milk powder in PBS-T containing $0.5 \%(\mathrm{w} / \mathrm{v})$ BSA. Plates were then co-incubated with $100 \mu \mathrm{l}$ per well each of HRP-labelled eqFg $\left(0.5 \mu \mathrm{g} \mathrm{ml}^{-1}\right)$ and serial dilutions $\left(0-10 \mu \mathrm{g} \mathrm{ml}^{-1}\right)$ of unlabelled eqFg, eqIgG4, eqIgG7 or eqIgG5. Plates were incubated for $45 \mathrm{~min}$ at room temperature and then developed with Sure Blue HRP substrate (KPL).

\section{RESULTS AND DISCUSSION}

\section{Residues 335-348 of FgBP are critical for IgG-Fc binding}

We previously showed, using a panel of N-terminal and Cterminal truncates and truncates lacking the repeat domains, that residues between the $\mathrm{A}$ and $\mathrm{B}$ repeats of 
$\mathrm{FgBP}$ (the $\mathrm{X}$ region in Fig. 1) are important for $\mathrm{IgG}-\mathrm{Fc}$ binding (Meehan et al., 2001). In order to identify which residues within this domain were critical for IgG binding, His-tagged recombinant FgBP proteins possessing internal amino acid deletions were constructed and purified (Figs $1 \mathrm{~b}$ and $2 \mathrm{a})$. FgBP1 was used as a starting point in construction, since it possesses similar IgG-Fc binding to that of wild-type FgBP purified directly from S. equi (Fig. 1a; Meehan et al., 2001).

In the design of recombinant derivatives, care was taken to ensure that the proposed deletions did not affect predicted
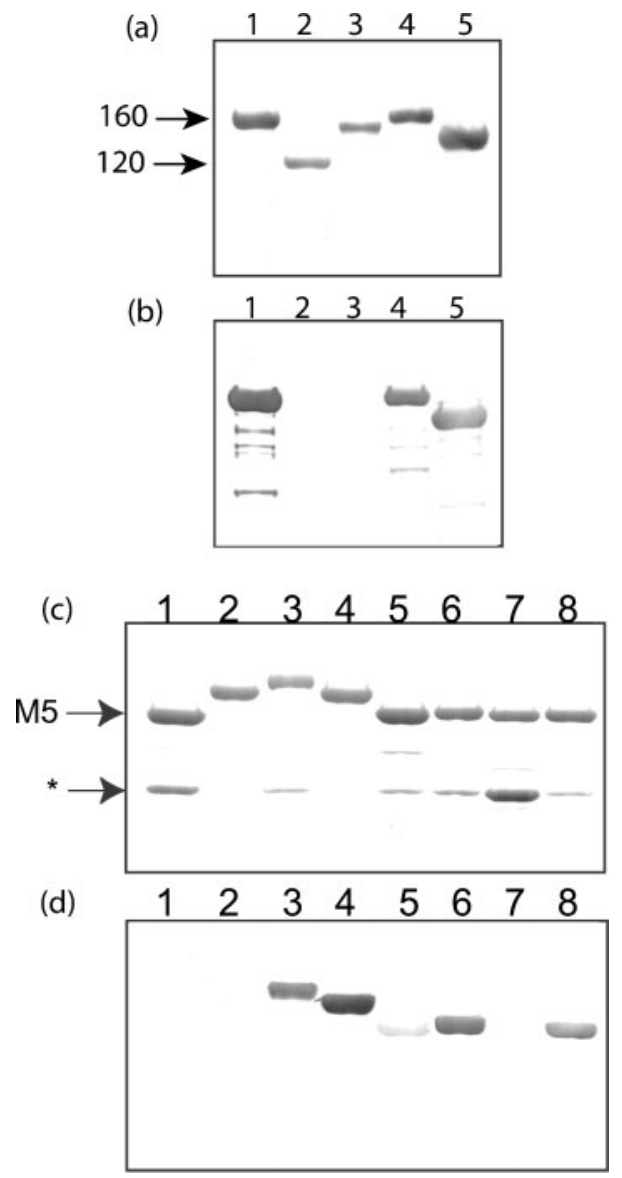

Fig. 2. SDS-PAGE and Western affinity blot analysis of purified His-tagged FgBP and M5FgBP proteins. (a) SDS-PAGE analysis of FgBP proteins. Lanes: 1, FgBP1; 2, FgBPX ${ }^{-}$; 3, FgBP18; 4, FgBP19; 5, FgBP20. Samples were analysed on a $7.5 \%(w / v)$ polyacrylamide separating gel. The positions of the 160 and $120 \mathrm{kDa}$ marker proteins are indicated. (b) An analogous Western affinity blot probed with HRP-conjugated eqlgG-Fc. (c) SDSPAGE analysis of M5FgBP proteins. Lanes: 1, M5; 2, M5FgBP2; 3, M5FgBP3; 4, M5FgBP7; 5, M5FgBP8; 6, M5FgBP9; 7, M5FgBP10; 8, M5FgBP12. Samples were analysed on a $12.5 \%$ $(\mathrm{w} / \mathrm{v})$ polyacrylamide separating gel. The positions of M5 and a degradation product (asterisk) seen with some of the recombinant proteins are indicated. (d) An analogous Western affinity blot probed with HRP-conjugated eqlgG-Fc. $\alpha$-helical coiled-coil structure as determined by the colLs program (Lupas et al., 1991; Lupas, 1996). Like FgBP1, these truncates lacked the signal sequence and the cell wall/ membrane-spanning region. In addition, they lacked residues $279-348$ that encompassed most of the $\mathrm{X}$ region between the $\mathrm{A}$ and $\mathrm{B}$ repeats $\left(\mathrm{FgBPX}^{-}\right)$or several shorter amino acid stretches within the $\mathrm{X}$ region that included residues 335-348 (FgBP18), residues 291-309 (FgBP19) and residues 309-330 (FgBP20; see Fig. 1b). Following analysis by SDS-PAGE, all purified proteins gave a single dominant band with an apparent $M_{\mathrm{r}}$ three to four times greater than that predicted from the sequence (see Fig. 2a). The aberrant mobility of $\mathrm{FgBP}$ and its recombinant truncates following SDS-PAGE is well documented (Meehan et al., 2000a, b).

Western affinity blots showed that the positive control (FgBP1), FgBP19 and FgBP20 all bound IgG-Fc, whereas FgBP18 and $\mathrm{FgBPX}^{-}$did not (Fig. 2b). To place these observations on a more quantitative basis and to relate them to previous experiments, IgG-Fc binding was analysed using semiquantitative dot blots. Results confirmed that FgBP18 and $\mathrm{FgBPX}^{-}$exhibited a dramatic (128-fold) reduction in binding to eqIgG-Fc and that FgBP19 and FgBP20 bound levels of eqIgG-Fc only slightly less (twofold to fourfold less) than that of FgBP1 (Fig. 3a). Furthermore, in ELISA tests, FgBP18 and FgBPX ${ }^{-}$failed to bind recombinant eqIgG4 and eqIgG7 subclasses. In contrast, eqIgG4 and eqIgG7 showed half-maximal binding of 0.5 and $0.8 \mu \mathrm{g} \mathrm{ml}^{-1}$ to FgBP1 and FgBP20, respectively (Fig. 4a, b). Analysis of the ability of these internally deleted FgBP truncates to bind Fg showed that FgBP18-20 bound the ligand with an avidity similar to that of FgBP1, whereas $\mathrm{FgBPX}^{-}$(with a larger deletion) possessed a fourfold reduction in Fg binding (Fig. 3b). Together, these results strongly suggest that residues 335-348 are crucial for IgG binding but not for Fg binding.

\section{Residues 329-360 of FgBP represent a minimal IgG-binding sequence}

Identification of critical IgG-binding residues allowed the rational design of experiments to identify a minimal IgGbinding sequence. Previously, circular dichroism and thermal stability analysis provided convincing evidence that the $\mathrm{C}$ terminus of $\mathrm{FgBP}$ encompassing the IgG-Fcbinding region contributes markedly to the thermal stability of $\mathrm{FgBP}$ and that coiled-coil structure may be required for ligand binding (Meehan et al., 2002). Therefore, to maintain the structural stability of short $\mathrm{FgBP}$ sequences and to minimize any loss in ligand binding resulting from destabilization of coiled-coil structure, M5FgBP chimeric proteins were constructed. M5 protein was employed as it possesses a high probability of $\alpha$-helical coiled-coil structure over the majority of the protein. M5 binds human Fg using residues in its B repeat region, but importantly does not bind IgG (Fig. 1c; Ringdahl et al., 2000). To construct M5FgBP chimeric proteins, the region 

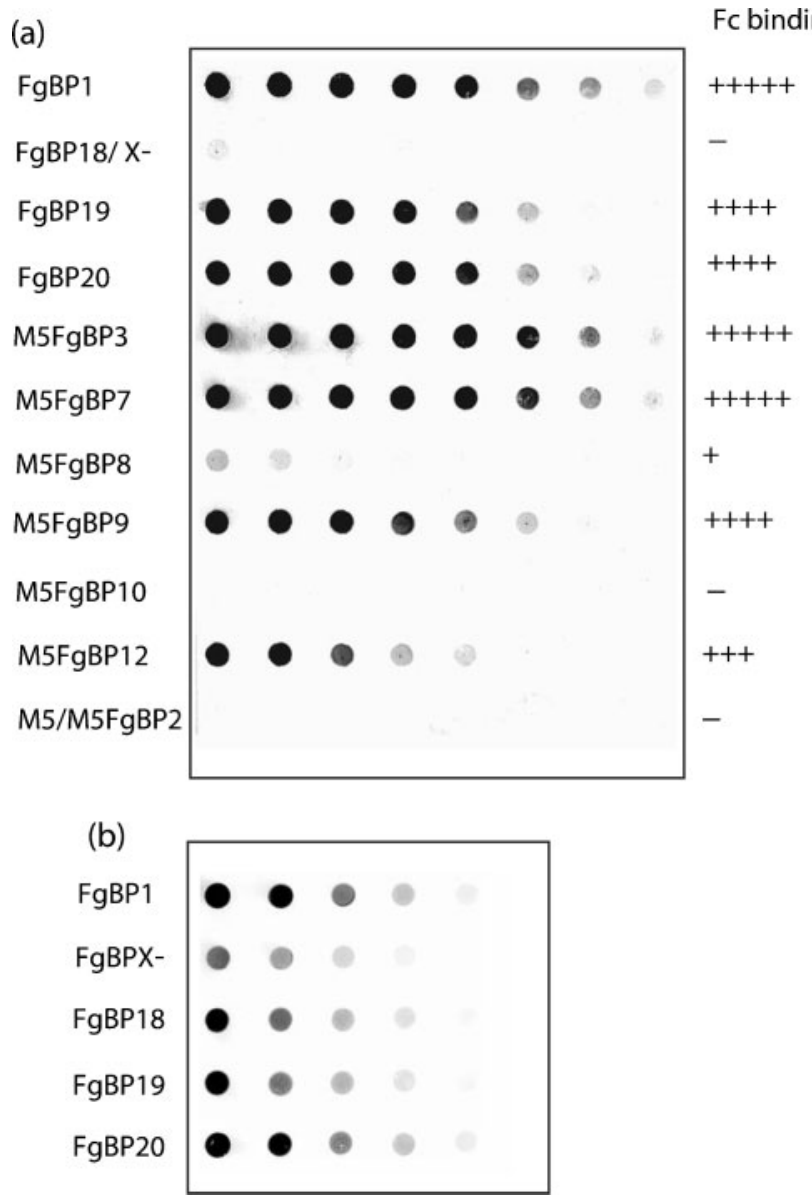

Fig. 3. (a) eqlgG-Fc and (b) Fg affinity dot blot analysis of undenatured FgBP truncates. Sample identity is indicated at the side of the blots. (a) The first well in each row of doubling dilutions contains 75 pmol of truncate and samples were probed with HRPconjugated eqlgG-Fc. FgBP truncates $X^{-}$and 18 gave identical negative results, as did M5, M5FgBP2 and M5FgBP10. IgG-Fc binding abilities are indicated to the right. Wild-type binding (equivalent to wild-type $\mathrm{FgBP}$ ) is indicated by +++++ , and twofold, fourfold to eightfold, 64-fold and 128-fold less binding than wild-type binding is indicated by,,++++++++ and - , respectively. (b) The first well in each row of doubling dilutions contains 9.4 pmol of truncate. Samples were probed with HRPconjugated eqFg.

of M5 DNA encoding the first 379 aa of mature M5 was first cloned into the expression vector pQE30. This recombinant plasmid expressed a truncated M5 protein which lacked the signal sequence and wall/membrane region, and was used as template for insertion of $f b p$ DNA. The resultant M5FgBP recombinant chimeras possessed FgBP sequences inserted between amino acids 360 and 361 of M5 protein (Fig. 1c), and included constructs that contained the $\mathrm{X}$ region $(\mathrm{M} 5 \mathrm{FgBP} 2)$ and the $\mathrm{X}$ region plus some downstream sequence (M5FgBP3 and M5FgBP7). Also constructed were several recombinants covering the region shown to be critical for IgG binding (M5FgBP8/9/
10/12; see Fig. 1b). Computer predictions showed that the coiled-coil structures of M5FgBP3 and M5FgBP8-10 were unchanged from that predicted for wild-type FgBP (Lupas et al., 1991; Lupas, 1996). These constructs were designed so that when fused into the M5 protein, FgBP residues would have the same predicted position in the heptad of coil-coil structure as in the native protein (Fig. 5a). Thus, Ala329 and Glu336 are at b positions, and Leu359 and Ser366 are at $\mathbf{d}$ positions. At either end of inserted $\mathrm{FgBP}$ sequences, positions $\mathbf{g}$ and $\mathbf{a}$ are provided by Asp and Ile, and positions $\mathbf{e}$ and $\mathbf{f}$ are provided by Glu and Leu from introduced restriction sites. For M5FgBP2, there is a predicted three-residue deletion (stutter) in the heptad repeat after Gly352 (d position) to Lys353 (a position). For M5FgBP7 and M5FgBP12, possession of Gln360 results in a predicted extra residue at the e position at the end of the FgBP insert. Deletions/insertions of these types can cause local aberrations in coiled-coil structure (Mason \& Arndt, 2004). However, all purified M5FgBP fusion proteins (Figs $1 \mathrm{~b}$ and $2 \mathrm{c}$ ) bound human $\mathrm{Fg}$, suggesting that no gross structural changes in M5 had occurred as a result of inclusion of FgBP sequences (data not shown).

Western IgG-affinity blotting experiments showed that M5, M5FgBP2, M5FgBP8 and M5FgBP10 bound eqIgG-Fc weakly or not at all and that the remaining chimeric proteins (M5FgBP3, 7, 9 and 12) bound detectable levels of eqIgG-Fc (Figs $1 \mathrm{~b}$ and $2 \mathrm{~d}$ ). IgG-affinity dot blots performed using undenatured recombinant chimeras confirmed and extended these observations (Fig. 3a). M5FgBP3 and M5FgBP7 bound wild-type levels of IgG-Fc, whereas M5FgBP9 (possessing residues 329-366 of FgBP) exhibited a slightly reduced (twofold) binding similar to that observed for FoBP19/20. M5FgBP12 (possessing residues 329-360 of FgBP) showed a further two- to fourfold reduction in binding. In contrast, M5FgBP2, M5FgBP8 (possessing residues 329-359 of FgBP) and FgBP10 (possessing residues 336-359 of FgBP) exhibited significantly reduced binding to IgG-Fc (Fig. 3a). ELISAs performed using recombinant eqIgG4 and eqIgG7 further revealed that: (a) M5FgBP3 bound similar levels of IgG to that of FgBP1; (b) M5FgBP9 and M5FgBP12 bound levels which were reduced compared with FgBP1 and somewhat similar to those observed for FgBP20; (c) M5 and M5FgBP10 did not bind either IgG subclass.

Taken together, these data suggest that the smallest region of FgBP that still retains significant IgG binding corresponds to residues 329-360 and encompasses the 14residue critical domain (residues 335-348) identified through the use of recombinant truncates. Gln360 appears to be crucial for IgG binding, since M5FgBP8 (residues 329-359) and M5FgBP2 (residues 273-356) show significantly reduced binding to IgG-Fc compared with M5FgBP12 (residues 329-360) and M5FgBP7 (residues 273-360). Residues 329-360 impart significant but not full wild-type binding to the $\mathrm{M} 5 \mathrm{FgBP}$ chimera. Available evidence suggests that upstream residues are necessary to achieve this. Firstly, M5FgBP7, which possesses residues 273-360, bound wild-type levels of IgG. Furthermore, 

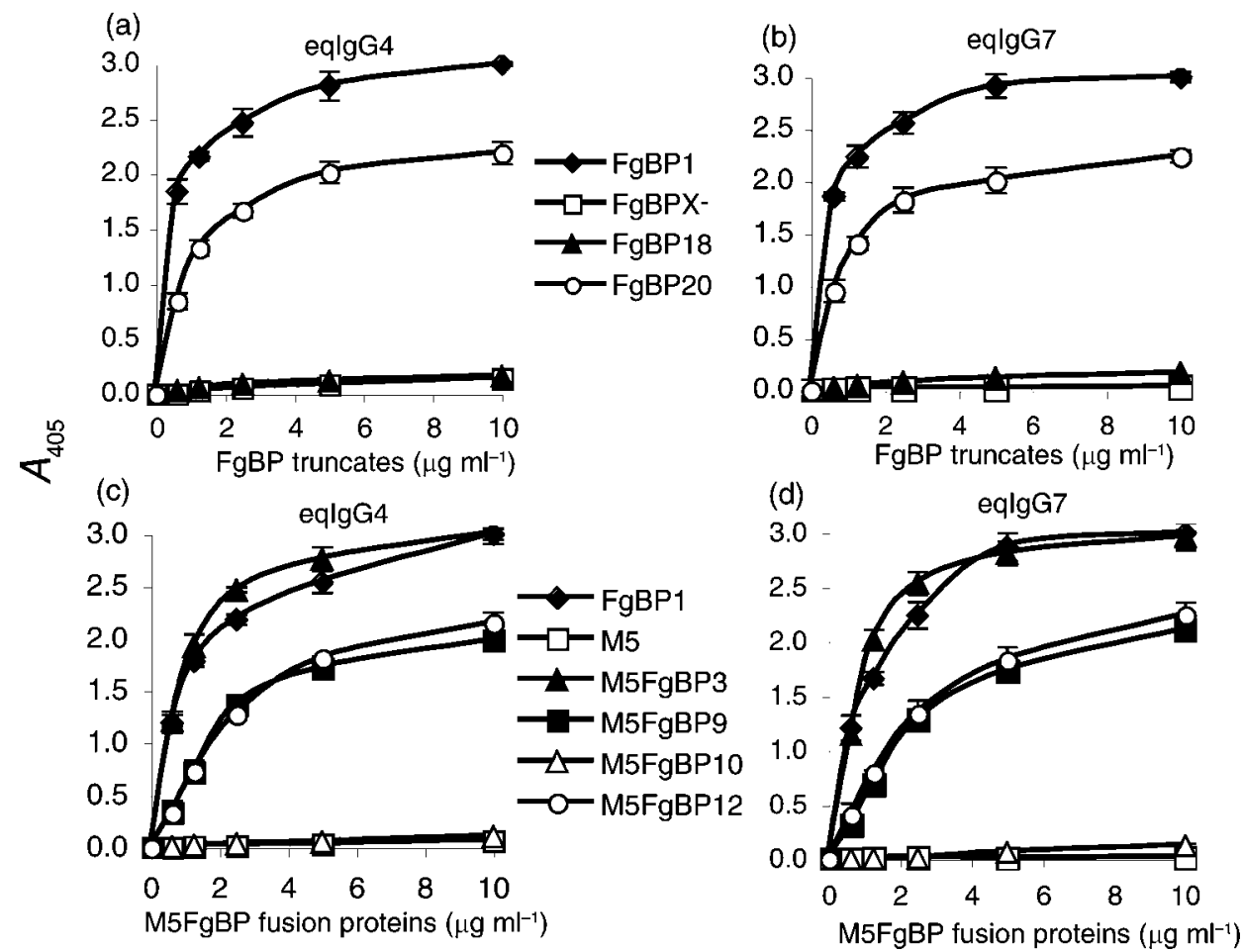

Fig. 4. ELISA analysis of the interaction of recombinant eqlgG4 and eqlgG7 with FgBP truncates $(a, b)$ and M5FgBP chimeric proteins $(c, d)$. The means ( $\pm S E)$ of three independent experiments are shown.

FgBP19 and FgBP20, which both possess deletions upstream of the 'critical' domain, exhibited a reduction in binding to eqIgG similar to that observed for the M5FgBP9/12 (Figs 3a and 4a, b). These upstream residues may provide some initial contact binding or play other structural role(s) which facilitate ligand interaction for the $\mathrm{M} 5 \mathrm{FgBP} 3$ and M5FgBP7 chimeras. A similar phenomenon may account for the significant reduction in IgG binding observed for truncate FgBP17, which lacks the first 182 residues of mature FgBP (Meehan et al., 2001).

The 32-residue IgG-binding domain possesses a strong probability of coiled-coil dimer formation. However, to date there is poor understanding of the structural parameters of streptococcal coiled-coil $\mathrm{M}$ or M-like proteins that are involved in IgG binding. Nonetheless, provided there is no local unwinding of the coiled-coil structure of $\mathrm{FgBP}$, residues occupying solvent exposedpositions (i.e. b, c, e, $\mathbf{f}$ and $\mathbf{g}$ ) are more likely to be involved in direct binding to IgG than non-polar residues buried at the interface of the two helices ( $\mathbf{a}$ and $\mathbf{d}$ positions; Fig. 5a).

\section{The IgG-binding domain of FgBP exhibits similarity to other coiled-coil and IgG-binding proteins}

With a view to identifying possible conserved residues or motifs involved in IgG binding to FgBP, BLAST homology searches were conducted using the C-terminal region (residues 273-482) of FgBP as the target. Proteins exhibiting homology with the IgG-binding domain of FgBP included several streptococcal proteins with predicted coiled-coil structure, some of which have been documented to bind IgG (Fig. 5b). These included SzM of S. equi subsp. zooepidemicus, Spa of S. pyogenes strain 18 and DemA/B of Streptococcus dysgalactiae, as noted elsewhere (Dale et al., 1999; Kelly et al., 2006; McLellan et al., 2001; Vasi et al., 2000). Others included (A repeats of) the Mrp/FcrA protein family of $S$. pyogenes and (repeated residues in) Sez 1778 of S. zooepidemicus strain MGCS10565 (Beres et al., 2008; O'Toole et al., 1992). Alignments of the above proteins revealed two conserved lysines corresponding to Lys337 and Lys353 of FgBP. Lys353 is followed by the sequence REIAEL, present as conserved substitutions in all proteins except Sez, which possesses Lys instead of Leu. Other residues in FgBP with conserved substitutions in homologous proteins include Glu334, Glu341, Ala343, Ileu349 and Ser351 present in or adjacent to the critical domain.

The ligand-binding properties of the proteins indicated in Fig. 5 have only been established for FgBP, DemA and the Mrp proteins, all of which bind Fg and IgG. Significantly, the IgG-binding domain of FgBP aligns with domains within Mrp and DemA previously implicated in IgG binding (Heath et al., 1990; Vasi et al., 2000). Although the ligand-binding properties of SzM, Spa and Sez 1778 are 
(a)

(b)
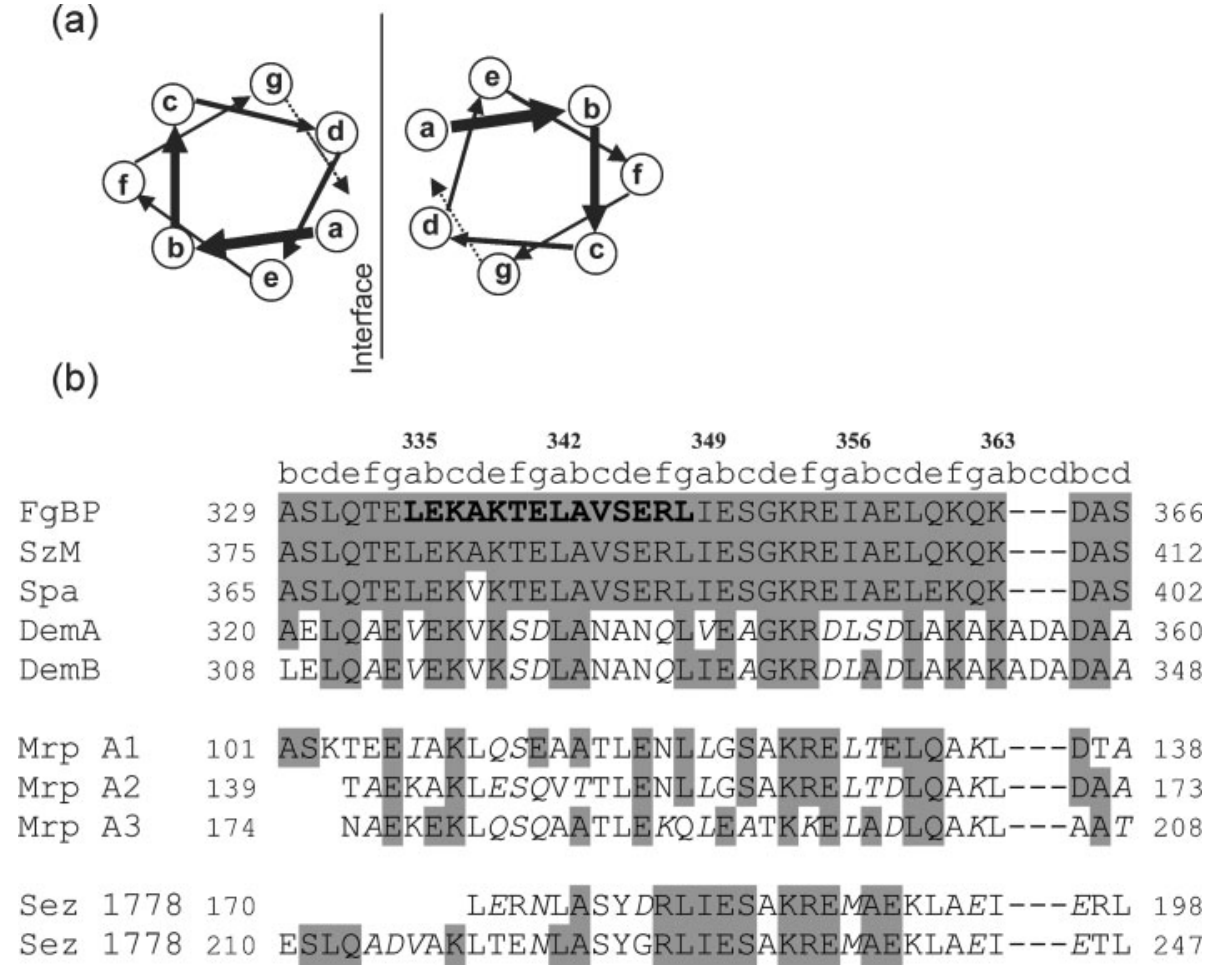

Fig. 5. Helical wheel representation of a dimeric coiled coil (a) and CLUSTAL $W$ alignment of amino acids 329-366 of FgBP with relevant positions in SzM, Spa, DemA, DemB and repeated sequences in Mrp and Sez 1778 (b). (a) Coiled-coil dimers consist of a heptad repeat pattern $(\mathbf{a}-\mathbf{g})$. $\mathbf{a}$ and $\mathbf{d}$ are typically hydrophobic core residues present at the interface of the two helices and are involved in dimer stabilization, whereas the remaining residues are generally hydrophilic. (b) Residues in FgBP shown to be critical for IgG binding are in bold type. Above the protein alignment are the predicted positions (a-g) of the amino acid residues in the heptad repeat of their coiled coil and the amino acid numbers of FgBP residues corresponding to the a position of heptad repeats. The Dem proteins possess a three-residue (bcd) insert in the heptad, which is indicated. Conserved residues are shaded grey and conserved substitutions indicated in italic type. The gene accession numbers for Spa, Sez 1778, DemA, DemB and Mrp4 are AAD42939, CPOO1129, CAB65411, CAB65413 and AAA26930, respectively.

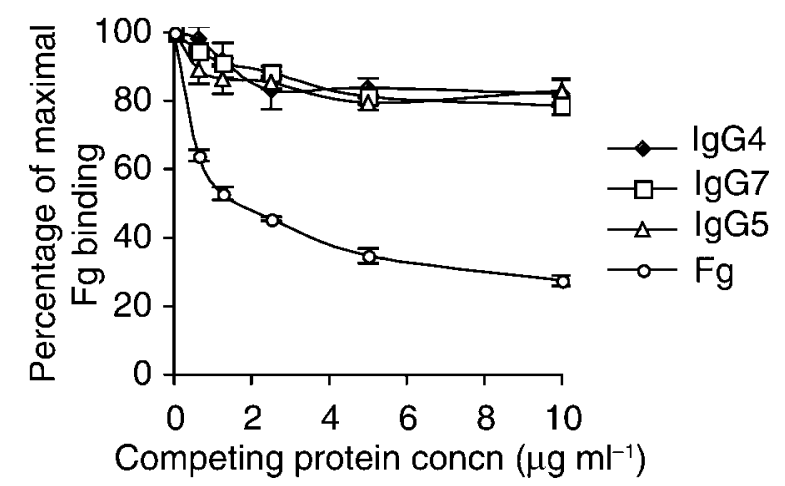

Fig. 6. ELISA of competition for binding to immobilized FgBP between HRP-labelled eqFg and increasing concentrations of unlabelled eqFg, recombinant eqlgG4, eqlgG7 or eqlgG5. The means $( \pm \mathrm{SE})$ of three independent experiments are shown. unknown (Beres et al., 2008; Dale et al., 1999; McLellan et al., 2001), it seems highly likely that Spa and SzM are capable of binding IgG given the virtual identity of their Cterminal domains to residues 255-534 of FgBP (95 and $98 \%$ identity, respectively; Dale et al., 1999; Kelly et al., 2006; McLellan et al., 2001) and that this accounts for the reported anti-phagocytic properties of Spa (Dale et al., 1999).

FgBP does not show homology to the proposed EQ-rich IgG3- and IgG1234-binding domains present in the class II group of $\mathrm{M}$ proteins and protein $\mathrm{H}$ of $S$. pyogenes (Bessen et al., 1997; Pack et al., 1996), nor does it show sequence homology to the IgG-binding domains of protein A or protein G. Despite the fact that all three proteins possess (some) $\alpha$-helical structure in their binding domains, available evidence indicates that the strategy used by FgBP for binding IgG-Fc differs from the mode of interaction adopted by protein $A$ and protein $G$. However, competition ELISAs and site-directed mutagenesis studies show that the FgBP-binding site on IgG 
overlaps with the binding sites of protein A and protein $G$ and involves residues in the Leu251-Ser254 and Leu432Tyr436 loops of IgG that interact with protein A and/or protein G (Lewis et al., 2008a). It is known that acidic and charged residues play important roles in the interaction between protein A/G and IgG-Fc (Sauer-Eriksson et al., 1995). One of the most critical interactions for protein $G$ is the one between the Leu251-Ser254 loop of IgG-Fc and Glu27, the carboxylate of Glu27 being held in position by hydrogen bonding to the adjacent Lys31 of protein G (Sauer-Eriksson et al., 1995; Sloan \& Hellinga, 1999). It has not escaped our notice that within the critical domain and adjacent to each other on the coiled-coil dimeric model structure of FgBP are residues Glu341 (g position), Lys337 (c position) and Lys339 (e position), which may perform analogous roles. However, it should be acknowledged that oppositely charged residues commonly occur in $\mathbf{e}$ and $\mathbf{g}$ positions of coiled-coils, providing inter-helical stability (Mason \& Arndt, 2004).

\section{IgG binding does not inhibit Fg binding}

In the current study, we have shown that IgG binding is critically dependent on a region (residues 335-348) distinct from the N-terminally located Fg-binding domain (Meehan et al., 2000a) and that deletion of the former region does not cause a significant reduction in Fg binding (Fig. 3b). In order to assess whether IgG binding could compromise $\mathrm{Fg}$ binding, competition ELISA tests were performed (Fig. 6). These tests showed that binding of eqFg to $\mathrm{FgBP}$ was slightly reduced in the presence of eqIgG4 and eqIgG7. However, this decrease was no greater than that shown in the presence of eqIgG5 (Fig. 6) or eqIgG3 (data not shown), both of which are eqIgG subclasses known to lack FgBP binding (Lewis et al., 2008a). In contrast, unlabelled eqFg could significantly compete with HRPeqFg for binding and HRP-eqFg binding was inhibited in a concentration-dependent manner by $70 \%$. Reverse competitive ELISAs showed that increasing doses of unlabelled Fg did not inhibit IgG binding (data not shown). Furthermore, preliminary surface plasmon resonance assays supported these findings and showed that Fg and IgG can bind simultaneously to FgBP (M. J. Lewis, unpublished observation).

\section{Concluding remarks}

An IgG-binding domain of FgBP has been identified which shows homology with regions present in other streptococcal coiled-coil proteins. Like FgBP, some of these proteins (Mrp and DemA) bind Fg and IgG. Future site-directed mutagenesis studies targeting conserved residues within this domain should allow identification of residues that are crucial for IgG binding. The IgG-binding domain of FgBP is located in a region of the protein different from that of the Fg-binding domain, and ELISA studies have indicated that binding of the two ligands is not competitive. Having defined the ligand-binding domains of FgBP, it is apparent that the protein can be separated into two halves, the $\mathrm{N}$ terminal Fg-binding region that is variable both within $S$. equi and among sub(species) (Anzai et al., 2005; Chanter et al., 2000; Kelly et al., 2006) and the conserved C-terminal region that harbours the IgG-binding domain. The conservation of this latter region suggests that it is not a target for strong immune or host-specific pressure. Other workers have suggested that acquisition and/or modification of the N-terminal region of FgBP was a key event in the evolution of $S$. equi from an ancestral S. zooepidemicus strain (Kelly et al., 2006; Waller \& Jolley, 2007). Whether this is solely related to its Fg-binding function or to another as yet unknown function remains to be determined.

FgBP is clearly an important anti-phagocytic protein of $S$. equi able to circumvent the alternative and classical complement pathways through its ability to bind $\mathrm{Fg}$ and IgG (Boschwitz \& Timoney, 1994a, b; Chanter et al., 2000; Meehan et al., 2001; Lewis et al., 2008a). Perhaps full resistance to phagocytosis is through cooperation between the two ligand-binding functions, as proposed for M22 protein, which binds C4b-binding protein and IgA-Fc (Carlsson et al., 2003). Certainly, the identification of IgGand Fg-binding sequences of $\mathrm{FgBP}$ should allow the rational construction of $S$. equi mutants of value in determining the relative contribution to $S$. equi virulence of FgBP-mediated $\mathrm{Fg} / \mathrm{IgG}$ binding and of other S. equi $\mathrm{Fg}$ and IgG-binding proteins (e.g. EAG and SzPSe) (Flock et al., 2004; Timoney et al., 1997).

\section{REFERENCES}

Anzai, T., Kuwamoto, Y., Wada, R., Sugita, S., Kakuda, T., Takai, S., Higuchi, T. \& Timoney, J. F. (2005). Variation in the N-terminal region of an M-like protein of Streptococcus equi and evaluation of its potential as a tool in epidemiologic studies. Am J Vet Res 66, 21672171.

Atkins, K. L., Burman, J. D., Chamberlain, E. S., Cooper, J. E., Poutrel, B., Bagby, S., Jenkins, A. T., Feil, E. J. \& van den Elsen, J. M. H. (2008). S. aureus IgG-binding proteins SpA and Sbi: host specificity and mechanisms of immune complex formation. Mol Immunol 45, 1600-1611.

Beres, S. B., Sesso, R., Pinto, S. W. L., Hoe, N. P., Porcella, S. F., DeLeo, F. R. \& Musser, J. M. (2008). Genome sequence of a Lancefield group C Streptococcus zooepidemicus strain causing epidemic nephritis: new information about an old disease. PLoS One 3, e3026.

Bessen, D. E., Izzo, M. W., McCabe, E. J. \& Sotir, C. M. (1997). Twodomain motif for IgG-binding activity of group A streptococcal $\mathrm{emm}$ gene products. Gene 196, 75-82.

Björck, L., Kastern, W., Lindahl, G. \& Widebäck, K. (1987). Streptococcal protein G, expressed by streptococci or by Escherichia coli, has separate binding sites for human albumin and IgG. Mol Immunol 24, 1113-1122.

Boschwitz, J. S. \& Timoney, J. F. (1994a). Characterization of the antiphagocytic activity of equine fibrinogen for Streptococcus equi subsp. equi. Microb Pathog 17, 121-129.

Boschwitz, J. S. \& Timoney, J. F. (1994b). Inhibition of C3 deposition on Streptococcus equi subsp. equi by $\mathrm{M}$ protein: a mechanism for survival in equine blood. Infect Immun 62, 3515-3520. 
Bradford, M. M. (1976). A rapid and sensitive method for the quantitation of microgram quantities of protein utilizing the principle of protein-dye binding. Anal Biochem 72, 248-254.

Burton, D. R. (1985). Immunoglobulin G: functional sites. Mol Immunol 22, 161-206.

Carlsson, F., Berggård, K., Stålhammer-Carlemalm, M. \& Lindahl, G. (2003). Evasion of phagocytosis through cooperation between two ligand-binding regions in Streptococcus pyogenes M protein. J Exp Med 198, 1057-1068.

Chanter, N., Talbot, N. C., Newton, J. R., Hewson, D. \& Verheyen, K. (2000). Streptococcus equi with truncated M-proteins isolated from outwardly healthy horses. Microbiology 146, 1361-1369.

Dale, J. B., Chiang, E. Y., Liu, S., Courtney, H. S. \& Hasty, D. L. (1999). New protective antigen of group A streptococci. J Clin Invest 103, 1261-1268.

DeLano, W. L., Ultsch, M. H., de Vos, A. M. \& Wells, J. A. (2000). Convergent solutions to binding at a protein-protein interface. Science 287, 1279-1283.

Flock, M., Jacobsson, K., Frykberg, L., Hirst, T. R., Franklin, A., Guss, B. \& Flock, J. I. (2004). Recombinant Streptococcus equi proteins protect mice in challenge experiments and induce immune response in horses. Infect Immun 72, 3228-3236.

Frick, I.-M., Wikström, M., Forsén, S., Drakenberg, T., Gomi, H., Sjöbring, U. \& Björck, L. (1992). Convergent evolution among immunoglobulin G-binding bacterial proteins. Proc Natl Acad Sci U S A 89, 8532-8536.

Goward, C. R., Scawen, M. D., Murphy, J. P. \& Atkinson, T. (1993). Molecular evolution of bacterial surface proteins. Trends Biochem Sci 18, 136-140.

Harrington, D. J., Sutcliff, I. C. \& Chanter, N. (2002). The molecular basis of Streptococcus equi infection and disease. Microbes Infect $\mathbf{4}$, 501-510.

Heath, D. G., Boyle, M. D. \& Cleary, P. P. (1990). Isolated DNA repeat region from $f(r A 76$, the Fc-binding protein gene from an M-type 76 strain of group A streptococci, encodes a protein with Fc-binding activity. Mol Microbiol 4, 2071-2079.

Johnsson, E., Berggård, K., Kotarsky, H., Hellwage, J., Zipfel, P. F., Sjöbring, U. \& Lindahl, G. (1998). Role of the hypervariable region in streptococcal M proteins: binding of a human complement inhibitor. J Immunol 161, 4894-4901.

Kelly, C., Bugg, M., Robinson, C., Mitchell, Z., Davis-Poynter, N., Newton, J. R., Jolley, K. A., Maiden, M. C. J. \& Waller, A. S. (2006). Sequence variation of the SeM gene of Streptococcus equi allows discrimination of the source of strangles outbreak. J Clin Microbiol 44, 480-486.

Laemmli, U. K. (1970). Cleavage of structural proteins during the assembly of the head of bacteriophage T4. Nature 227, 680-685.

Lewis, M. J., Meehan, M., Owen, P. \& Woof, J. M. (2008a). A common theme in interaction of bacterial immunoglobulin-binding proteins with immunoglobulins illustrated in the equine system. J Biol Chem 283, 17615-17623.

Lewis, M. J., Wagner, B. \& Woof, J. M. (2008b). The different effector function capabilities of the seven equine IgG subclasses have implications for vaccine strategies. Mol Immunol 45, 818-827.

Lupas, A. (1996). Prediction and analysis of coiled-coil structures. Methods Enzymol 266, 513-525.

Lupas, A., Van Dyke, M. \& Stock, J. (1991). Predicting coiled coils from protein sequences. Science 252, 1162-1164.

Mason, J. M. \& Arndt, K. M. (2004). Coiled-coil domains: stability, specificity, and biological implications. ChemBioChem 5, 170-176.
McLellan, D. G. J., Chiang, E. Y., Courtney, H. S., Hasty, D. L., Wei, S. C., Hu, M. C., Walls, M. A., Bloom, J. J. \& Dale, J. B. (2001). Spa contributes to the virulence of type 18 group A streptococci. Infect Immun 69, 2943-2949.

Meehan, M., Nowlan, P. \& Owen, P. (1998). Affinity purification and characterization of a fibrinogen-binding protein complex which protects mice against lethal challenge with Streptococcus equi subsp. equi. Microbiology 144, 993-1003.

Meehan, M., Muldowney, D. A., Watkins, N. J. \& Owen, P. (2000a). Localization and characterization of the ligand-binding domain of the fibrinogen-binding protein (FgBP) of Streptococcus equi subsp. equi. Microbiology 146, 1187-1194.

Meehan, M., Muldowney, D. A., O'Meara, F. \& Owen, P. (2000b). Neither the A- nor B-repeat regions of the fibrinogen-binding protein of Streptococcus equi subsp. equi are essential for fibrinogen binding. FEMS Microbiol Lett 190, 317-321.

Meehan, M., Lynagh, Y., Woods, C. \& Owen, P. (2001). The fibrinogen-binding protein $(\mathrm{FgBP})$ of Streptococcus equi subsp. equi additionally binds IgG and contributes to virulence in a mouse model. Microbiology 147, 3311-3322.

Meehan, M., Kelly, S. M., Price, N. C. \& Owen, P. (2002). The Cterminal portion of the fibrinogen-binding protein of Streptococcus equi subsp. equi contains extensive $\alpha$-helical coiled-coil structure and contributes to thermal stability. FEMS Microbiol Lett 206, 81-86.

Moks, T., Abrahmsén, L., Nilsson, B., Hellman, U., Sjöquist, J. \& Uhlén, M. (1986). Staphylococcal protein A consists of five IgGbinding domains. Eur J Biochem 156, 637-643.

Navarre, W. W. \& Schneewind, O. (1999). Surface proteins of Grampositive bacteria and mechanisms of their targeting to the cell wall envelope. Microbiol Mol Biol Rev 63, 174-229.

O'Toole, P., Stenberg, L., Rissler, M. \& Lindahl, G. (1992). Two major classes in the M protein family in group A streptococci. Proc Natl Acad Sci U S A 89, 8661-8665.

Pack, T. D., Podbielski, A. \& Boyle, M. D. (1996). Identification of an amino acid signature sequence predictive of protein G-inhibitable IgG3-binding activity in group-A streptococcal IgG-binding proteins. Gene 171, 65-70.

Pleass, R. J., Areschoug, T., Lindahl, G. \& Woof, J. M. (2001). Streptococcal IgA-binding proteins bind in the $\mathrm{C} \alpha 2-\mathrm{C} \alpha 3$ interdomain region and inhibit binding of IgA to human CD89. J Biol Chem 276, 8197-8204.

Ringdahl, U., Svensson, H. G., Kotarsky, H., Gustafsson, M., Weineisen, M. \& Sjöbring, U. (2000). A role for the fibrinogenbinding regions of streptococcal $\mathrm{M}$ proteins in phagocytosis resistance. Mol Microbiol 37, 1318-1326.

Sambrook, J., Fritsch, E. F. \& Maniatis, T. (1989). Molecular Cloning: a Laboratory Manual, 2nd edn. Cold Spring Harbor, NY: Cold Spring Harbor Laboratory.

Sauer-Eriksson, A. E., Kleywegt, G. J., Uhlén, M. \& Jones, T. A. (1995). Crystal structure of the C2 fragment of streptococcal protein $G$ in complex with the Fc domain of human IgG. Structure 3, 265-278.

Sheoran, A. S., Sponseller, B. T., Holmes, M. A. \& Timoney, J. F. (1997). Serum and mucosal antibody isotype response to M-like protein (SeM) of Streptococcus equi in convalescent and vaccinated horses. Vet Immunol Immunopathol 59, 239-251.

Sloan, D. J. \& Hellinga, H. W. (1999). Dissection of the protein G B1 domain binding site for human IgG Fc fragment. Protein Sci 8, 1643-1648.

Timoney, J. F. (1993). Strangles. Vet Clin North Am Equine Pract 9 , 365-374.

Timoney, J. F. (2004). The pathogenic equine streptococci. Vet Res 35, 397-409. 
Timoney, J. F., Artiushin, S. C. \& Boschwitz, J. S. (1997). Comparison of the sequences and functions of Streptococcus equi M-like proteins SeM and SzPSe. Infect Immun 65, 3600-3605.

Uhlén, M., Guss, B., Nilsson, B., Gatenbeck, S., Philipson, L. \& Lindberg, M. (1984). Complete sequence of the staphylococcal gene encoding protein A. A gene evolved through multiple duplications. J Biol Chem 259, 1695-1702.
Vasi, J., Frykberg, L., Carlsson, L. E., Lindberg, M. \& Guss, B. (2000). M-like proteins of Streptococcus dysgalactiae. Infect Immun 68, 294-302.

Waller, A. S. \& Jolley, K. A. (2007). Getting a grip on strangles: recent progress towards improved diagnostics and vaccines. Vet J 173, 492501.

Edited by: H. Ingmer 